\title{
Avaliação da influência da utilização de agregado miúdo reciclado em argamassas estabilizadas
}

\author{
Evaluation of the influence of the use of recycled fine \\ aggregate in stabilised mortars
}

\section{Thiago da Silva Santana \\ Cláudio Henrique de Almeida Feitosa Pereira}

Resumo

omo forma de reduzir o volume descartado de resíduos da construção civil, o uso de agregado reciclado na produção de concretos e argamassas tem se mostrado uma opção sustentável. Dessa forma, o presente estudo tem como objetivo avaliar a influência da substituição de agregado miúdo natural por agregado miúdo reciclado nas propriedades das argamassas estabilizadas de revestimento. Para tal, foram elaboradas quatro argamassas estabilizadas, uma argamassa com agregado miúdo natural (areia lavada de leito de rio) e outras três argamassas com teores de substituição em massa de $25 \%$, 50\% e $100 \%$ de agregado miúdo natural por agregado miúdo reciclado. A avaliação das argamassas estabilizadas de revestimento foi realizada tanto no estado fresco (massa específica, índice de consistência, penetração de cone) como no estado endurecido (densidade no estado endurecido, resistência à tração na flexão e à compressão, absorção de água por capilaridade, módulo de elasticidade dinâmico). Por meio desses ensaios, verificou-se no estado fresco semelhança de comportamento entre a argamassa com agregado natural e as argamassas com agregado miúdo reciclado. Já no estado endurecido ocorreu redução das propriedades mecânicas e aumento da absorção de água das argamassas com o aumento do teor de substituição do agregado miúdo natural pelo agregado miúdo reciclado.

Palavras-chave: Resíduos de construção civil. Agregado miúdo reciclado. Argamassa estabilizada. Argamassa de revestimento.

\begin{abstract}
The use of recycled aggregate in the production of concrete and mortars has shown to be a sustainable way to reduce the volume of constructionwaste. Thus, this study aims to evaluate the influence of the substitution of natural fine aggregate by recycled fine aggregate on the properties of stabilised mortars. To this end, four stabilised mortars: one with natural aggregate (sand washed from the river bed) and three other mortars with $25 \%, 50 \%$ and $100 \%$ levels of

substitution of natural fine aggregate were recycled. The evaluation of stabilised mortars was performed in the fresh state (specific mass, consistency index, cone penetration) and in the hardened state (hardened density, flexural and compressive strength, water absorption by capillarity and dynamic modulus of elasticity). These testsshowed a similar behaviour, in the fresh state, between the mortar with natural aggregate and the mortars with recycled aggregate. In the hardened state, there was a reduction in the mechanical properties and an increase in the water absorption of the mortarswith a higherlevel of substitution of the natural fine aggregate by the recycled fine aggregate.

Keywords: Construction waste. Recycled fine aggregates. Stabilisedmortar. Coatingmortar.
\end{abstract}

${ }^{1}$ Thiago da Silva Santana 'Universidade de Brasília Brasília -DF - Brasil

${ }^{2}$ Cláudio Henrique de Almeida Feitosa Pereira 2Universidade de Brasília Brasília - DF - Brasil

Recebido em 25/09/19

Aceito em 04/04/20 


\section{Introdução}

A indústria da construção civil está entre uma das maiores consumidoras de matérias-primas naturais e geradoras de resíduos. Esse segmento é responsável pela produção de mais de 70 milhões de toneladas de resíduos por ano (CONTRERAS et al., 2016; PAZ; LAFAYETTE, 2016). Portanto, a indústria da construção civil pode ser considerada uma grande causadora de impacto ambiental.

Os resíduos da construção civil (RCC) são definidos como aqueles materiais oriundos de construções, reformas e demolições de obras de construção civil, assim como o material resultante da preparação e escavação de terrenos (CONSELHO..., 2002).

Esse material apresenta grande heterogeneidade devido ao fato de sua composição variar de acordo com a forma de geração (LLATAS, 2013; CONTRERAS et al., 2016; OLIVEIRA, 2017) e dos materiais empregados (MARTÍNEZ et al., 2013).

A utilização de processo de logística reversa nos RCC por meio da reciclagem como agregados se mostra uma opção para a redução de seu descarte, assim como da exploração de agregados naturais.

A parte desse material classificada como classe A (CONSELHO..., 2002) pode ser utilizada como agregado reciclado. A possibilidade dessa utilização é regulamentada pela NBR 15116 (ABNT, 2004), a qual permite a utilização dos agregados reciclados em pavimentação e concretos não estruturais. Ressalta-se que para a produção de argamassas ainda não existe norma brasileira específica.

A utilização dos agregados reciclados na produção de materiais cimentícios, como argamassas e concretos, vem se mostrando viável em diversas pesquisas. Trabalhos como Leite (2001), Pedrozo (2008), Lotfi et al. (2015) e Dimitriou, Savva e Petrou (2018) apresentam a possibilidade da utilização do agregado reciclado de RCC em concretos. Já as avaliações realizadas por Jiménez et al. (2013) e por Ledesma et al. (2016) relatam a possibilidade de substituição de até $40 \%$ e de até $50 \%$ respectivamente do agregado miúdo natural (AMN) pelo agregado miúdo reciclado (AMR) na elaboração de argamassas.

Os AMR apresentam características diferentes do AMN. Se comparados, os AMR apresentam forma mais angular e irregular, devido ao processo de britagem, e textura rugosa, em consequência da pasta de cimento aderida à superfície do agregado (SOLYMAN, 2005; SANTOS, 2016), maiores porosidades, devido à argamassa aderida ao material e à presença de impurezas leves (BUYLE-BODIN; HADJIEVAZAHARIEVA, 2002), assim como maior superfície específica (FUMOTO; YAMADA, 2002) e, em geral, menor massa específica (EVANGELISTA et al., 2015; MARTÍNEZ et al., 2013). Além disso, apresentam maior absorção de água, devido à porosidade da argamassa aderida ao agregado (SOLYMAN, 2005; EVANGELISTA et al., 2015; CORRÊEA; RÊGO; FEITOSA, 2018).

Essas características do AMR influenciam nas propriedades das argamassas tanto no estado fresco como no estado endurecido.

A elevada absorção de água dos agregados reciclados acarretará o aumento da porosidade e da absorção de água das argamassas com esse material, como verificado por Martínez et al. (2013). Essa característica também levará à redução da densidade aparente das argamassas nos estados fresco e endurecido, como relatado por Ledesma et al. (2016) para taxas de substituição de agregado natural por agregado reciclado superiores a 50\% e por Carasek et al. (2018) para argamassas com 100\% de agregados reciclados. Essa redução de densidade aparente também é atribuída à menor massa específica e à maior porosidade do agregado reciclado, assim como ao aumento da relação água-materiais secos das argamassas com agregado reciclado (CARASEK et al., 2018).

Além disso, a utilização do agregado miúdo pode gerar redução na trabalhabilidade das argamassas devido à absorção de água desses agregados, como verificado por Jimenez et al. (2013), com o teor de substituição de $40 \%$.

Quanto às resistências mecânicas, Leite et al. (2000) e Braga, De Brito e Veiga (2012) relatam aumentos nas resistências à tração na flexão e à compressão. Leite et al. (2000) associam essa maior resistência à redução da relação água-cimento(a/c) devido à absorção de água dos agregados reciclados. Já Corinaldesi e Moriconi (2009) e Fan et al. (2015) evidenciaram reduções nessa propriedade. Os autores associam tal redução à argamassa aderida no agregado reciclado.

O mesmo ocorre com o módulo de elasticidade. Braga, De Brito e Veiga (2012) relatam aumento do módulo de elasticidade, enquanto Leite et al. (2000) obtiveram redução do módulo. Leite et al. (2000) associam essa

306 Santana, T. da S.; Pereira, C. H. de A. F. 
redução à maior porosidade do agregado reciclado, o qual leva a argamassa a apresentar-se mais deformável se comparada à argamassa com agregado reciclado.

Entre as argamassas que poderiam fazer o uso de agregados reciclados há as argamassas estabilizadas. Essas argamassas apresentam período de utilização superior ao dasargamassas convencionais ou industrializadas devido à utilização do aditivo estabilizador de hidratação. Além disso, são produzidas em centrais de concreto e fornecidas no estado fresco, prontas para uso (BAUER et al., 2015; OLIVEIRA, 2017). Dependendo da dosagem deaditivo, o período de estabilização da argamassa estabilizada pode durar até $72 \mathrm{~h}$ (RIXOM; MAILVAGANAM, 1999).

Uma especificidade das argamassas estabilizadas é a recomendação de se utilizar uma lâmina d'água para a manutenção da umidade do material ao longo do período de estabilização. Porém, Casali et al. (2011), os quais avaliaram a utilização da lâmina de água de proteção em cinco lotes de argamassas estabilizadas, verificaram que as argamassas sem a proteção dessa lâmina apresentaram maiores resistências mecânicas, o que indica um possível aumento da relação a/c devido à utilização dessa lâmina.

$\mathrm{O}$ aditivo estabilizador de hidratação, usado nas argamassas estabilizadas, aumenta o tempo de aplicação do material devido a sua capacidade de retardar a hidratação dos silicatos tricálcicos $\left(\mathrm{C}_{3} \mathrm{~S}\right)$, dos silicatos dicálcicos $\left(\mathrm{C}_{2} \mathrm{~S}\right)$ e dos aluminatos tricálcicos $\left(\mathrm{C}_{3} \mathrm{~A}\right)$. Esse fenômeno ocorre devido ao fato de o aditivo impedir a nucleação dos C-S-H e CH ou retardar o crescimento desses produtos de hidratação, o que irá também desacelerar a hidratação do $\mathrm{C}_{3} \mathrm{~A}$ (PAOLINE; KHURANA, 1998).

A utilização das argamassas estabilizadas pode trazer vantagens no processo de execução de revestimentos de argamassa devido à retirada das etapas de dosagem e de mistura no canteiro de obra, o que leva ao aumento da produtividade. Além disso, a produção da argamassa em centrais dosadoras de concreto possibilita maior controle do material e, consequentemente, melhor qualidade (BAUER et al., 2015).

Comparando-se as propriedades das argamassas estabilizadas com as argamassas industrializadas e as produzidas em obras, Trevisol Junior (2015) verificou que a argamassa estabilizada manteve o nível de trabalhabilidade por um período maior, além de menor absorção de água e menor retração e expansão se comparada às demais argamassas.

Apesar da maior manutenção da trabalhabilidade e demais benefícios das argamassas estabilizadas, Bauer et al. (2015) ressaltam a falta de referência normativa no Brasil para a especificação, o uso e o emprego dessas argamassas, o que resulta na falta de controle técnico e na limitação de uso.

Assim, verifica-se que uma das alternativas para a destinação dos resíduos de construção civil é sua utilização como agregado reciclado em matrizes cimentícias. Entre as matrizes destacam-se as argamassas devido àpossibilidade de substituição de altos teores de agregado natural por agregados reciclados, como demonstrado por Ledesma et al. (2016) e Carasek et al. (2018). Ao mesmo tempo, há crescentes estudos acerca das argamassas estabilizadas, visando àmelhor compreensão desses materiais e de suas características.

Dessa forma, este estudo visa contribuir com essa lacuna por meio da análise da influência da substituição do AMN pelo AMR, produzido em usina de reciclagem de RCC no Distrito Federal, sobre as propriedades no estado fresco e no estado endurecido das argamassas estabilizadas.

\section{Procedimentos metodológicos}

\section{Materiais}

Os materiais utilizados na produção das argamassas foram cimento Portland de alta resistência inicial (CPVARI), agregado miúdo natural, agregado miúdo reciclado, água, aditivo estabilizante de hidratação (AEH) e aditivo incorporador de ar (IAR). O CPV-ARI utilizado possui massa específica de $3,10 \mathrm{~g} / \mathrm{cm}^{3}$, segundo a NBR NM 23 (ABNT, 2009a), tempo de início de pega de $130 \mathrm{~min}$, e de fim de pega de $210 \mathrm{~min}$, determinados de acordo com a NBR NM 65 (ABNT, 2009b). Os aditivos utilizados foram AEH Matchem AE 20 e IAR Matchem AE 10. De acordo com o ACI 212.3R-16 (AMERICAN..., 2016), os AEH são compostos de ácidos carboxílicos e sais de ácidos orgânicos contendo fósforo, e os IAR, de sais de resinas de madeira, alguns detergentes sintéticos, sais de sulfonados lignina, sais de ácidos de petróleo, alquilbenzeno sulfonatos, sais de hidrocarbonetos sulfonados e outras substâncias. 
Os aditivos foram caracterizados segundo os procedimentos da NBR 10908 (ABNT, 2008) sendo determinadosa massa específica, o teor de sólidos e o pH. Os resultados dessas determinações são apresentados nas Tabelas 1 e 2 .

O AMN utilizado na elaboração das argamassas estabilizadas foi uma areia lavada de rio, escolhida em razão de ser empregada na produção de argamassas estabilizadas comercializadas no Distrito Federal. Juntamente com o CPV-ARI, o AEH e o AIR foram disponibilizados por uma central produtora de argamassas estabilizadas do Distrito Federal. Já o AMR foi escolhido em virtude de sua disponibilidade para fornecimento regional, coletado diretamente emusina de reciclagem localizada em Sobradinho, DF. Esse agregado foi obtido por meio da britagem dos resíduos de construção civil, com a utilização de um britador de mandíbulas, com posterior lavagem do material para a retirada do material pulverulento.

Para a caracterização dos agregados foram realizados os ensaios de composição granulométrica (ABNT, 2003b); massa específica para agregados miúdos (ABNT, 1987); teor de material passante na peneira de $75 \mu \mathrm{m}$ (ABNT, 2003a); absorção de água para agregados miúdos (ABNT, 2000); torrões de argila e materiais friáveis (ABNT, 2010); e teor de cloretos e teor de sulfatos por cromatografia iônica. A técnica de cromatografia iônica foi utilizada em virtude de seu maior poder de detecção se comparada aos procedimentos apresentados pela NBR 9917 (ABNT, 2009c). Os resultados desses ensaios são apresentados na Tabela 3 e na Figura 1 .

Verifica-se em relação ao AMN que o AMR apresenta menor massa específica, maior teor de finos e maior absorção de água. Observa-se ainda que ambos os agregados estão em conformidade com os requisitos da NBR 15116 (ABNT, 2004), visto que apresentaram valores de teor de finos, absorção de água, teor de cloretos e teor de sulfatos inferiores aos limites estabelecidos. Ressalta-se que o teor de argila dos agregados foi superior ao limite determinado pela NBR 15116 (ABNT, 2004), porém para esta pesquisa esse teor acima do limite não foi considerado fator impeditivo para a produção de argamassas. Esse maior teor de finos poderá gerar influência no comportamento e nas propriedades das argamassas.

Tabela 1 - Aditivo incorporador de ar

\begin{tabular}{c|c|c}
\hline Propriedades & Resultados & Dados do fabricante $\left(\mathbf{2 5}^{\mathbf{0}} \mathbf{C}\right)$ \\
\hline Massa específica $\left(\mathrm{g} / \mathrm{cm}^{3}\right)$ & 1,01 & 0,99 a 1,03 \\
Teor de sólidos $(\%)$ & 5,3 & - \\
$\mathrm{pH}$ & 10,0 & 10,0 a 12,0 \\
\hline
\end{tabular}

Tabela 2 - Aditivo estabilizador de hidratação

\begin{tabular}{c|c|c}
\hline Propriedades & Resultados & Dados do fabricante $\left(\mathbf{2 5}^{\circ} \mathbf{C}\right)$ \\
\hline Massa específica $\left(\mathrm{g} / \mathrm{cm}^{3}\right)$ & 1,17 & 1,15 a 1,21 \\
Teor de sólidos $(\%)$ & 38,2 & - \\
pH & 5,6 & 6,0 a 8,0 \\
\hline
\end{tabular}

Tabela 3 - Características dos agregados

\begin{tabular}{l|c|c}
\hline \multicolumn{1}{c|}{ Propriedades } & Areia natural & Areia reciclada \\
\hline Dmáx (mm) & 0,6 & 4,8 \\
Módulo de finura & 1,27 & 1,83 \\
Massa específica $\left(\mathrm{g} / \mathrm{cm}^{3}\right)$ & 2,65 & 2,54 \\
Massa unitária $\left(\mathrm{kg} / \mathrm{dm}^{3}\right)$ & 1,84 & 1,77 \\
Teor de finos $(\%)$ & 1,58 & 9,04 \\
Absorção de água (\%) & 0,44 & 5,48 \\
Teor de argila (\%) & 6,93 & 5,13 \\
Cloretos (\%) & 0,00015 & 0,00010 \\
Sulfatos (\%) & 0,00019 & 0,01030 \\
\hline
\end{tabular}

308 Santana, T. da S.; Pereira, C. H. de A. F. 
Figura 1 - Curvas granulométricas

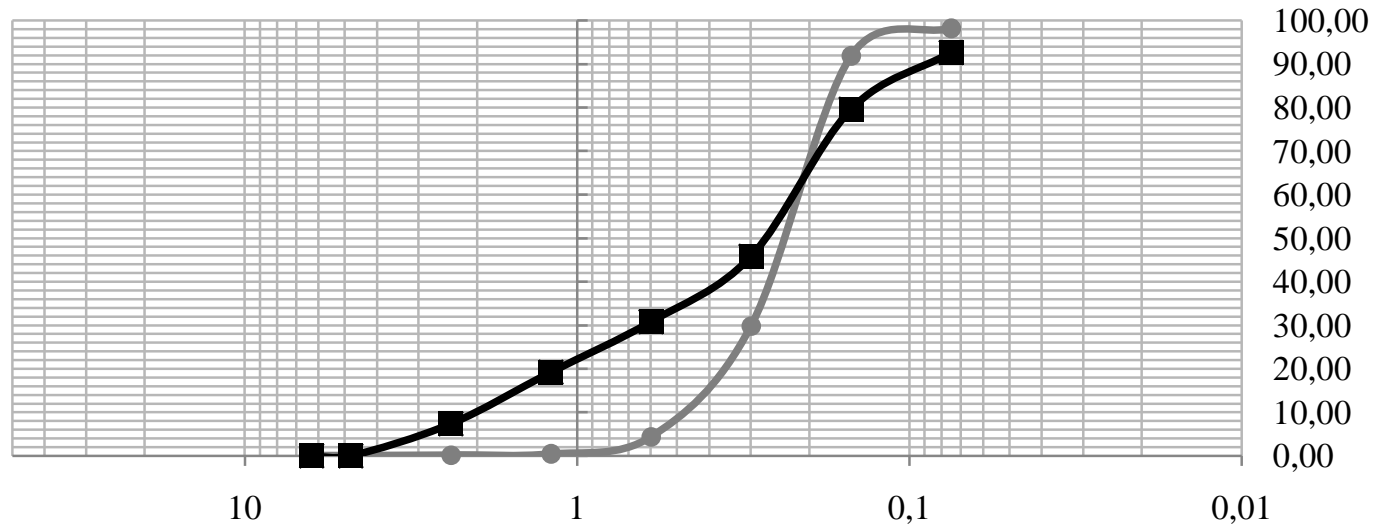

Abertura (mm)

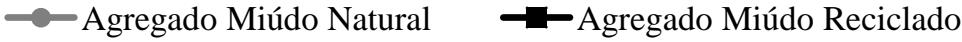

Também foi utilizada para a produção das argamassas estabilizadas a água fornecida pela rede de abastecimento do Distrito Federal (Caesb).

\section{Métodos}

Foram produzidas quatro argamassas, uma com AMN (AAMN), duas com teores de substituição de $25 \%$ (AAMR25\%) e 50\% (AAMR50\%), e uma com substituição total do AMN pelo AMR (AAMR100\%). As substituições das diferentes porcentagens de AMN por AMR foram realizadas, em massa, nas frações retidas nas peneiras com abertura de 2,36 mm, 1,18 mm, $600 \mu \mathrm{m}, 300 \mu \mathrm{m}, 150 \mu \mathrm{m}, 75 \mu \mathrm{m}$ e fundo, visando evitar modificações na curva granulométrica de referência (AMN). Devido àmenor massa específica do agregado reciclado, se comparado ao agregado natural, da ordem de $0,10 \mathrm{~g} / \mathrm{cm}^{3}$, ocorrerá o acréscimo de um volume maior de agregados reciclados se comparado ao volume dos agregados naturais.

As quatro argamassas foram preparadas pelo Método de Rápido de Ajuste (MRA), desenvolvido no Laboratório de Ensaio de Materiais da Universidade de Brasília (LEM/UnB) e utilizado em suas rotinas de estudos em argamassas, como nos trabalhos de Oliveira (2017) e Santana (2018). O MRA consiste nos seguintes procedimentos:

(a) executar mistura manual dos insumos anidros separadamente;

(b) colocar todos os insumos anidros na cuba do misturador;

(c) executar mistura mecânica em velocidade baixa, adicionando $50 \%$ da água de mistura ao longo de $30 \mathrm{~s}$;

(d) executar mistura mecânica por $30 \mathrm{~s}$ em velocidade baixa;

(e) desligar o misturador e realizar a raspagem da superfície interna da cuba e da pá;

(f) deixar a argamassa em repouso por $90 \mathrm{~s}$;

(g) adicionar o restante da água (50\%) juntamente com o aditivo estabilizador de hidratação e o aditivo incorporador de ar, os quais devem ser previamente misturados; e

(h) executar mistura mecânica na velocidade baixa por $60 \mathrm{~s}$.

Foi predefinida uma consistência inicial das argamassas baseada no ensaio de penetração de cone. Para se obter essa consistência foi realizada a compensação de $80 \%$ da água absorvida pelo AMR e adicionada água de amassamento até se atingir a consistência predeterminada de $(75 \pm 5) \mathrm{mm}$ de penetração de cone.

O tempo de estabilização das argamassas especificado foi de $8 \mathrm{~h}$, visto que esse período abrange a jornada de trabalho de um dia. Dessa forma, a dosagem do $\mathrm{AEH}$, teor em relação à massa de cimento, visou obter uma consistência das argamassas com $8 \mathrm{~h}$ de estabilização dentro da faixa de $(55 \pm 5) \mathrm{mm}$. O teor de IAR foi 
padronizado em $0,2 \%$ da massa de cimento. Dessa forma, as argamassas apresentaram os traços demonstrados na Tabela 4.

Ressalta-se que as dosagens dos aditivos seguiram as recomendações do fabricante (Matchem), sendo de $0,2 \%$ a $1,0 \%$ para o IAR e de $0,4 \%$ a $1,5 \%$ para o AEH.

Para a avaliação das argamassas estabilizadas com diferentes teores de substituição, foram realizados no estado fresco os ensaios de determinação da densidade de massa e teor de ar incorporado (ABNT, 2005a), índice de consistência (ABNT, 2016), penetração de cone (AMERICAN..., 2014) eretenção de água (ABNT, 2005b). Já no estado endurecido determinaram-se a densidade de massa no estado endurecido (ABNT, 2005c), a resistência à tração na flexão e à compressão (ABNT, 2005d), o módulo de elasticidade dinâmico (AMERICAN..., 2009) e a absorção de água por capilaridade e coeficiente de capilaridade (ABNT, 2005f).

\section{Resultados e discussões}

Nesta seção são apresentados os resultados obtidos nos ensaios na avaliação das argamassas com AMN e diferentes teores de AMR e suas respectivas análises.

\section{Densidade e teor de ar incorporado}

Os resultados dos ensaios de densidade e teor de ar incorporado realizados no estado fresco são apresentados na Figura 2.

Tabela 4 - Traço das argamassas

\begin{tabular}{l|c|c|c}
\hline \multicolumn{1}{c|}{ Argamassa } & $\begin{array}{c}\text { Traço unitário, em massa } \\
(\mathbf{c}: \mathbf{a n}: \mathbf{a r}: \mathbf{a} / \mathbf{c})\end{array}$ & $\begin{array}{c}\text { AEH } \\
(\boldsymbol{\%})\end{array}$ & $\begin{array}{c}\text { IAR } \\
(\boldsymbol{\%})\end{array}$ \\
\hline AAMN & $1: 6,0: 0,0: 1,2$ & 0,4 & 0,2 \\
AAMR25\% & $1: 4,5: 1,5: 1,4$ & 0,4 & 0,2 \\
AAMR50\% & $1: 3,0: 3,0: 1,6$ & 0,7 & 0,2 \\
AAMR100\% & $1: 0,0: 6,0: 1,9$ & 1,0 & 0,2 \\
\hline
\end{tabular}

Nota: *cimento : areia natural : areia reciclada : a/c.

Figura 2 - Comparativo da densidade no estado fresco e o teor de ar incorporado vs teor de substituição do agregado

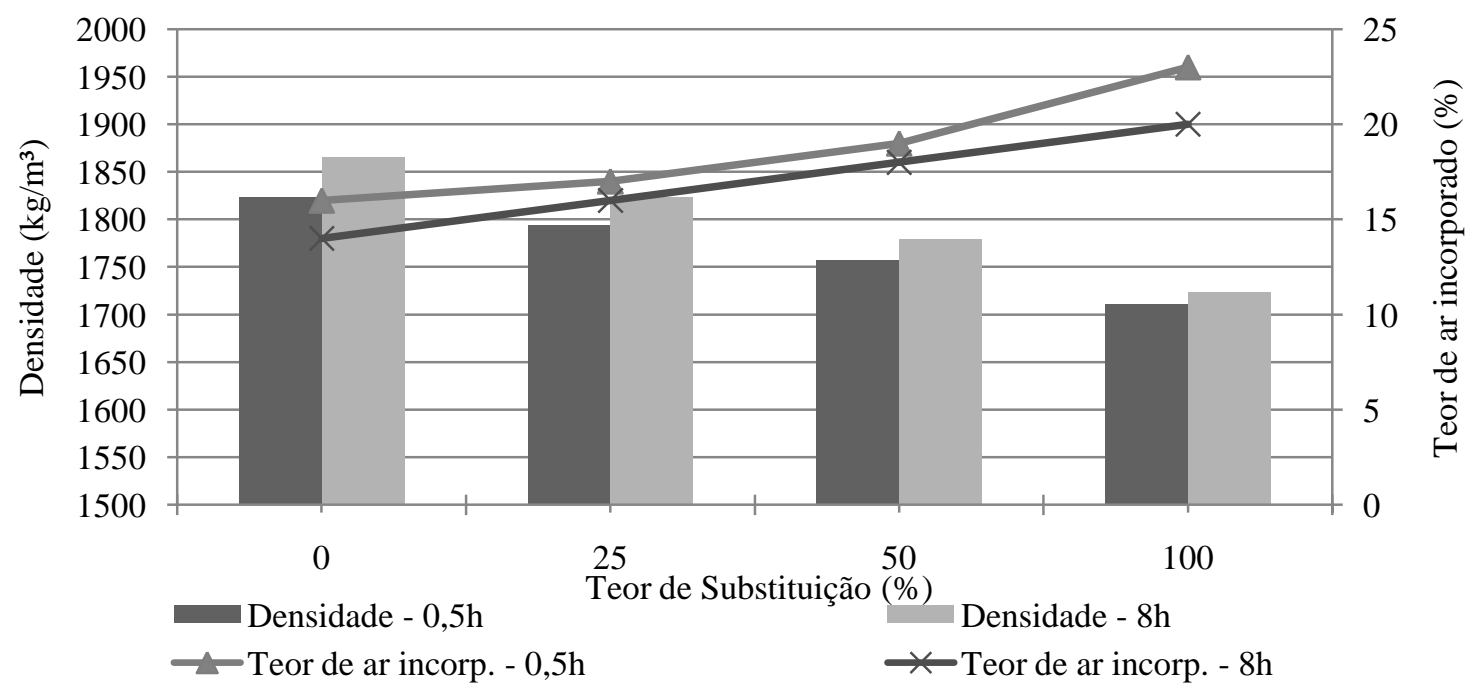

310 Santana, T. da S.; Pereira, C. H. de A. F. 
As massas específicas das argamassas reduziram gradativamente com o aumento do teor de substituição das areias. Essa redução deve-se à menor massa específica do AMR, ao aumento dos teores de água nos traços devido à substituição da AMN pelo AMR e ao aumento do teor de ar, como observado na Figura 2. Essa redução também foi identificada nas argamassas avaliadas por Jiménez et al. (2013) para teores de substituição de AMN por AMR superiores a 25\%. Ledesma et al. (2016) identificaram esse comportamento com teores de substituição superiores a 50\%, e Carasek et al. (2018) com a utilização de teores de $100 \%$ de AMR.

A AAMN e a AAMR25\% se enquadraram na classe D5 da NBR 13281 (ABNT, 2005e);já as demais argamassas se enquadraram na classe D4.

Observa-se ainda que as quatro argamassas apresentaram redução do teor de ar incorporado ao final das $8 \mathrm{~h}$ de estabilização, se comparadoaos valores obtidos $0,5 \mathrm{~h}$ após a preparação das argamassas. Consequentemente, ocorreu aumento da densidade das argamassas ao final das $8 \mathrm{~h}$ de estabilização.

\section{Índice de consistência e penetração de cone}

As argamassas com AMR e a AAMN apresentaram valores de espalhamento e de penetração de cone semelhantes depois de $0,5 \mathrm{~h}$ e de $8 \mathrm{~h}$. Essa semelhança, nesta pesquisa, está associada à fixação da consistência por meio da penetração de cone, tanto no início como no final do tempo de estabilização das argamassas. Nota-se ainda ganho de consistência nas quatro argamassas, visto que os valores de espelhamento e de penetração de cone com $8 \mathrm{~h}$ foram menores aos obtidos com $0,5 \mathrm{~h}$.

Na Figura 3 são apresentados os resultados dos ensaios de índice de consistência e penetração de cone.

\section{Retenção de água}

Os resultados do ensaio de retenção de água são apresentados na Tabela 5.

Independentemente dos teores de substituição, as quatro argamassas apresentaram valores de retenção de água semelhantes no intervalo de $60 \%$ a $66 \%$. Dessa forma, as quatro argamassas enquadram-se na classe U1 (<78\%), de acordo com a NBR 13281 (ABNT, 2005e). Essa semelhança provavelmente se devea um equilíbrio entre o aumento da relação a/c, do ar incorporado, os quais reduziriam a retenção de água, e do teor de finos, aumentando a retenção de água das argamassas com agregado miúdo reciclado.

Figura 3 - Comparativo índice de consistência e penetração de cone vs teor de substituição do agregado

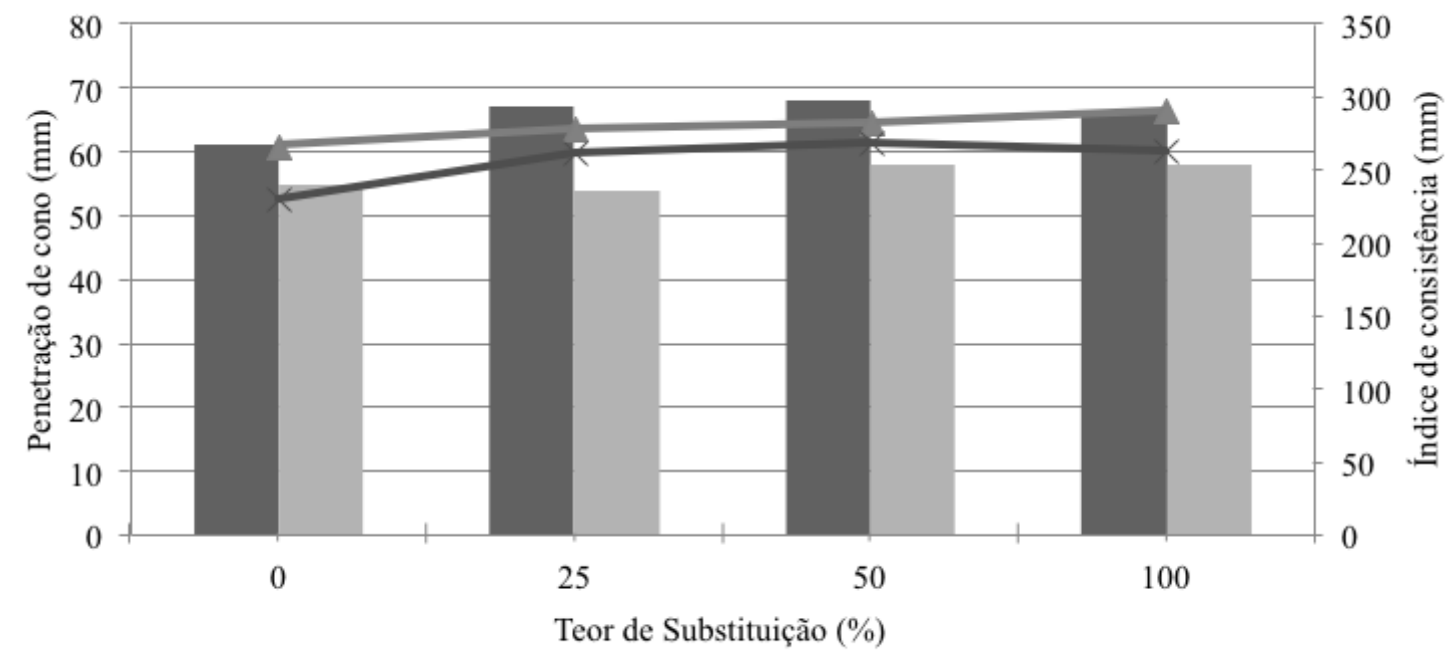

Penet. de cone $0,5 \mathrm{~h} \rightleftharpoons$ Penet. de cone $-8 \mathrm{~h} \leftarrow$ Índice de consist. - $0,5 \mathrm{~h} \leftarrow$ Índice de consist. $8 \mathrm{~h}$

Tabela 5 - Resultados do ensaio de retenção de água

\begin{tabular}{c|c|c|c|c}
\hline Ensaio & AAMN & AAMR25\% & AAMR50\% & AAMR100\% \\
\hline Retenção de água & 66 & 60 & 61 & 64 \\
\hline
\end{tabular}




\section{Densidade e coeficiente de capilaridade}

Os resultados dos ensaios de densidade e coeficiente de capilaridade realizados no estado endurecido são apresentados nas Figura 4.

As densidades das argamassas no estado endurecido se reduziram com o aumento dos teores de substituição das areias, assim como ocorreu no estado fresco. Essa redução está associada ao aumento da porosidade ocasionada devido aos aumentos do teor de água e do teor de ar incorporado, além da menor massa específica do agregado miúdo reciclado em relação ao agregado miúdo natural. Esse aumento da porosidade se reflete no aumento dos coeficientes de capilaridade com o aumento dos teores de substituição.

A redução da densidade com o aumento do teor de substituição das areias foi observada por Samie et al. (2015) e Pimentel et al. (2018) na avaliação de argamassas com AMR.

Em relação à classificação da NBR 13281 (ABNT, 2005e), a AAMN enquadrou-se nas classes M5 e C4 em relação respectivamente à densidade e ao coeficiente de capilaridade. As AAMR25\% eAAMR50\% se enquadraram nas classes M4 e C5. Já a AAMR100\% se enquadrou nas classes M3 e C6.

\section{Resistência à tração na flexão e à compressão}

Os resultados dos ensaios de resistência à tração na flexão e à compressão realizados no estado endurecido são apresentados nas Figuras 5 e 6.

Ocorreu redução gradativa da resistência à tração na flexão e à compressão com o aumento dos teores de substituição das areias. Essa redução da resistência à compressão deve-se ao aumento do teor de água e, consequentemente, ao aumento da relação a/c e ao teor de ar incorporado das argamassas com o incremento dos teores de substituição das areias. As AAMN e AAMR25\% enquadraram-se nas classes R3 e P5, a AAMR50\% nas classes R3 e P4, e a AAMR100\% nas classes R2 e P3.

Essa redução da resistência à tração na flexão das argamassas com AMR também foi identificada por Pimentel et al. (2018) em argamassas mistas e hidráulicas com teores de substituição de 30\% e $60 \%$.

Corinaldesi e Moriconi (2009), na avaliação de argamassas com 100\% de agregado miúdo reciclado de concreto, e Fan et al. (2015), com a substituição do AMN pelo AMR de concreto em teores de 25\%, 50\% e $100 \%$, também identificaram redução da resistência à compressão nas argamassas com AMR.

Figura 4 - Comparativo densidade e coeficiente de capilaridade vs teor de substituição do agregado

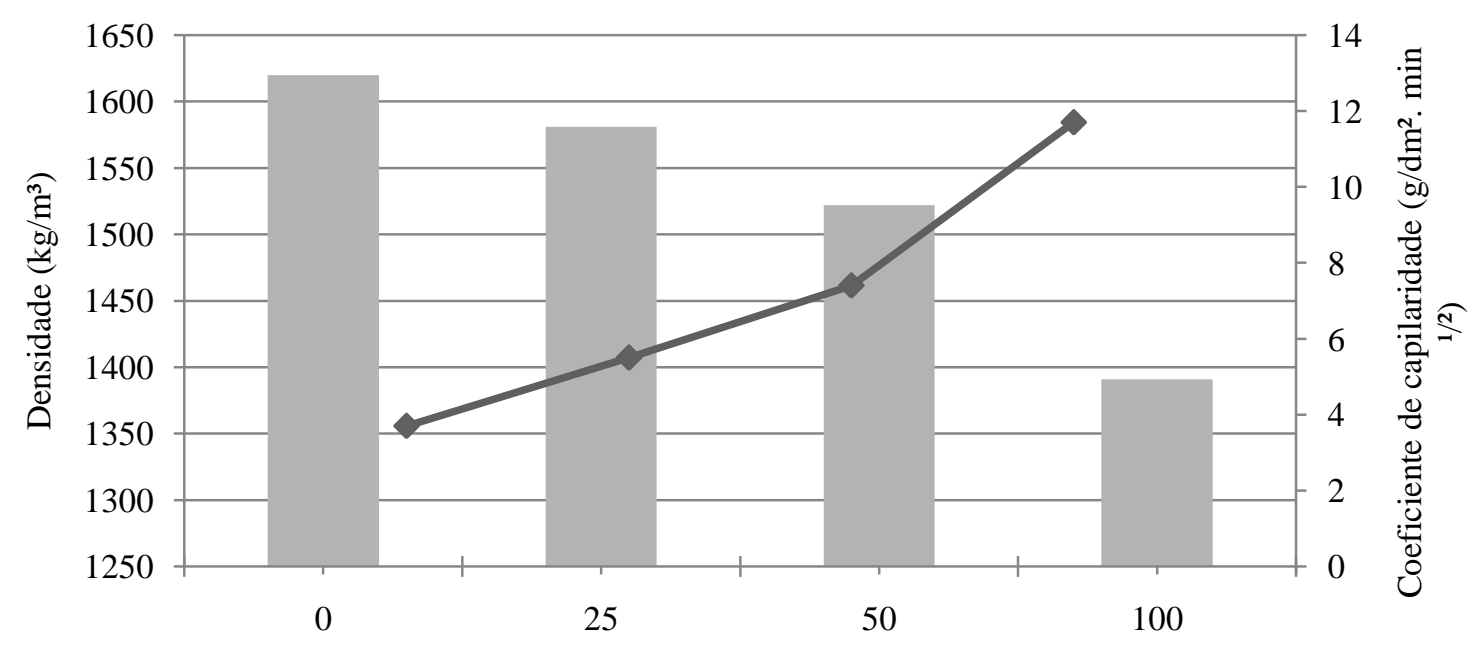

Teor de Substituição (\%)

Densidade $\quad \sim$ Coeficiente de capilaridade 
Figura 5 - Comparativo resistência à tração média na flexão vsteor de substituição do agregado

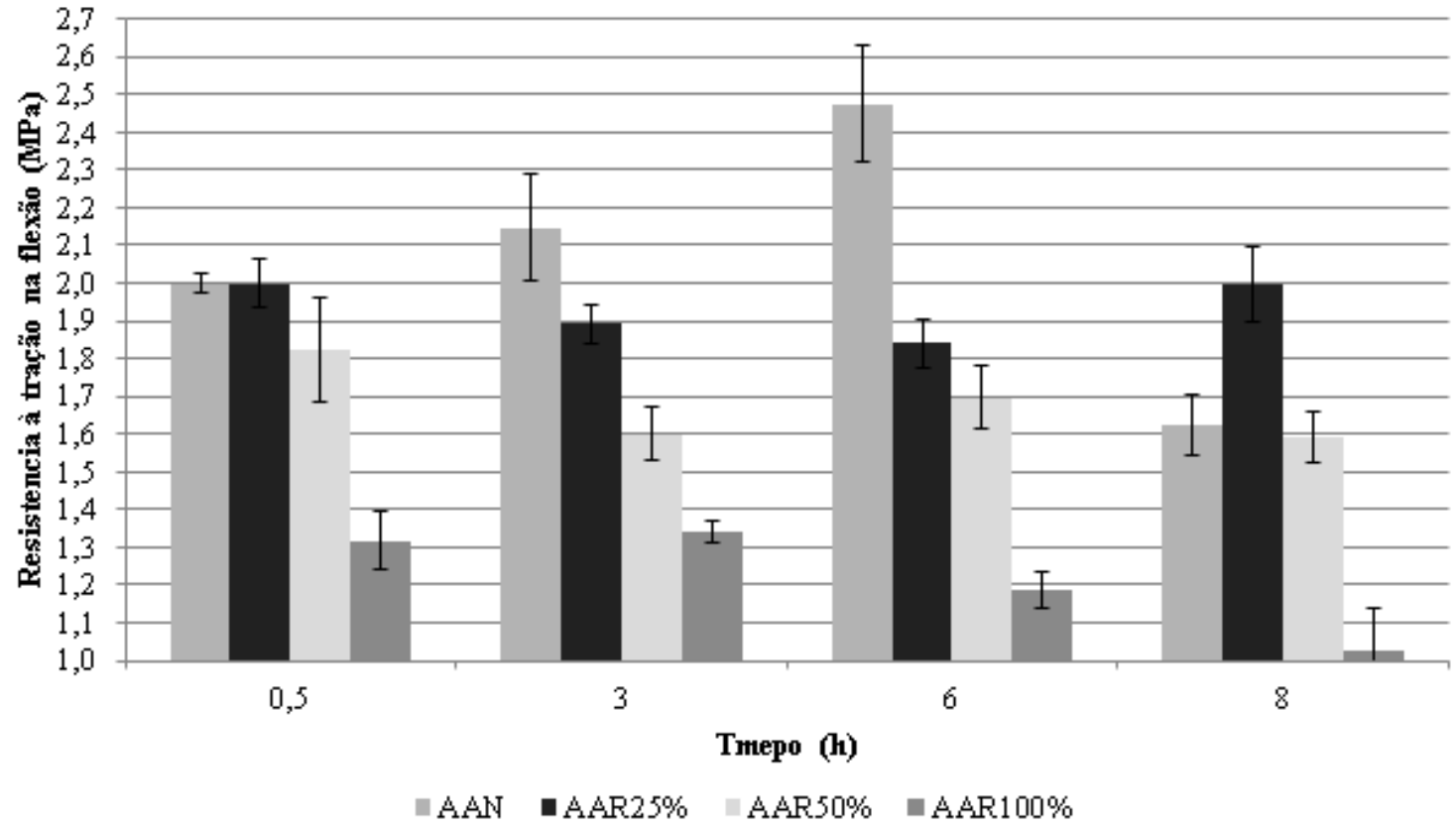

Figura 6 - Comparativo resistência à compressão média vs teor de substituição do agregado

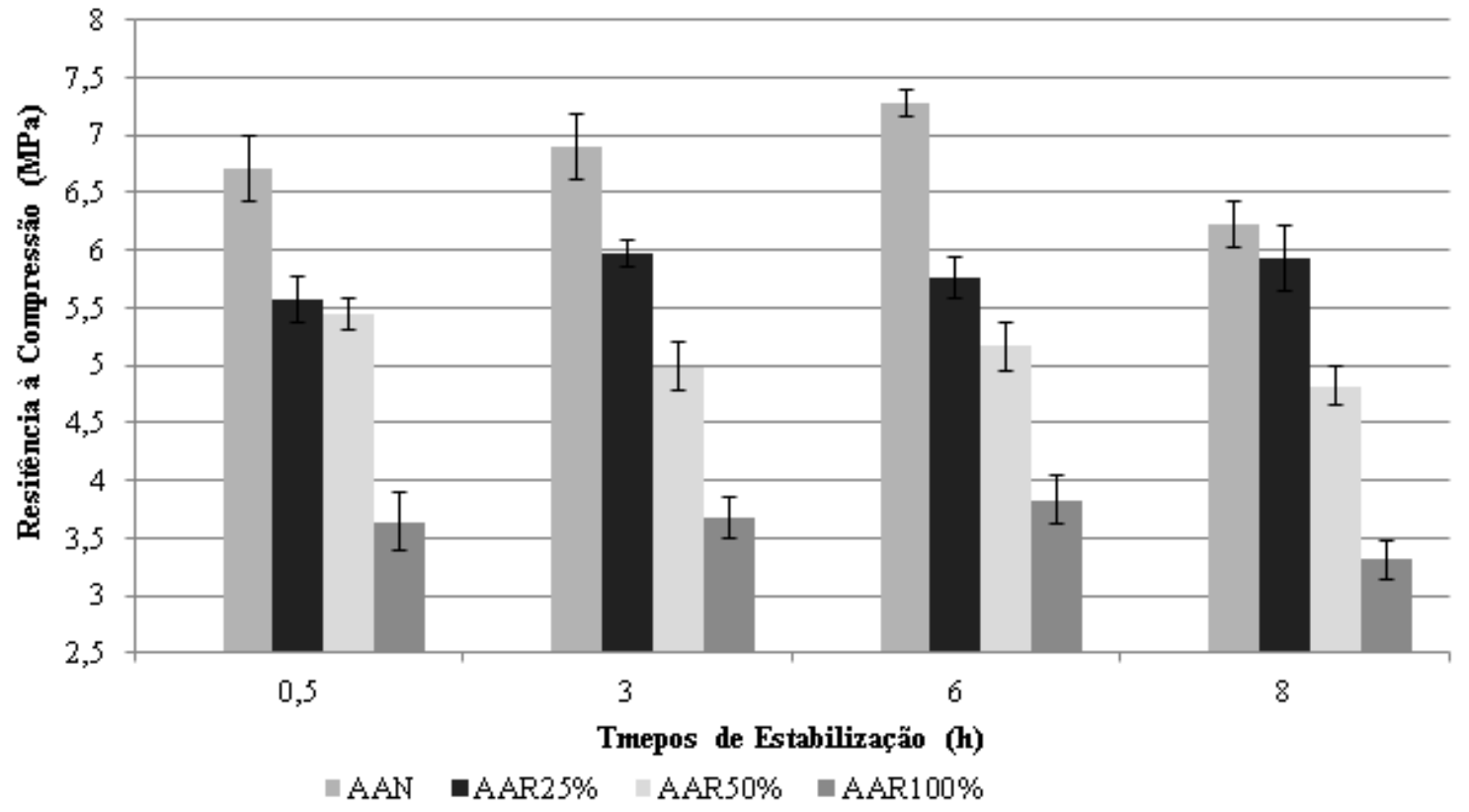

Ressalta-se que, apesar de as argamassas não terem apresentado variação nos valores de resistência à tração na flexão e de resistência à compressão nos diferentes tempos analisados, cada uma das argamassas manteve a mesma classificação nos tempos de $0,5 \mathrm{~h}, 3 \mathrm{~h}, 6 \mathrm{~h}$ e $8 \mathrm{~h}$.

Para verificar a significância dos resultados de resistência à tração na flexão e de resistência à compressão, os quais são apresentados nas Tabelas 6 e 7, foi realizada análise de variância ANOVA.

$\mathrm{Na}$ análise ANOVA, para a variação ser significativa, o p-valor não deve ser maior que o nível de significância (0,05). Verifica-se nas Tabelas 6 e 7 que os valores de p-valor tanto para a resistência à tração na flexão quanto para a resistência à compressão foram inferiores a 0,05 . 
Dessa forma, houve diferença significativa na resistência à tração na flexão e na resistência à compressão entre as argamassas com diferentes teores de substituição de AMN por AMR.Essa diferença entre as argamassas com diferentes teores de substituição fica evidente no Teste de Duncan (Tabelas 8 e 9).

Nas Tabelas 8 e 9 pode-se verificar por meio dos resultados do Teste de Duncan que foram formados quatro grupos distintos quanto aos valores de resistência à tração na flexão e na resistência à compressão. Essa formação de grupos distintos de acordo com o teor de substituição dos agregados indica que a substituição do AMN pelo AMR acarretou redução gradativa da resistência à tração na flexão e da resistência à compressão com o aumento do teor de substituição.

\section{Módulo de elasticidade dinâmico}

Na Tabela 10 são apresentados os resultados com os valores médios obtidos no ensaio de determinação do módulo de elasticidade.

Nota-se redução do módulo de elasticidade dinâmica com o aumento do teor de substituição das areias. Essa redução se deve ao aumento da relação a/c ocasionado pelo aumento do teor de água necessário para a elaboração das argamassas com AMR. Leite et al. (2000) atribuem a redução do módulo de elasticidade das argamassas com AMR à maior porosidade desse agregado, que torna a argamassa mais deformável.

Tabela 6 - Análise ANOVA - Resistência à tração na flexão das AAMN e AAMR

\begin{tabular}{c|c|c|c|c|c}
\hline Fonte da variação & SQ & MQ & F & P- valor & F crítico \\
\hline Resistência à tração na flexão & 1912676,079 & 956338 & 92,90257 & $2,77 \mathrm{E}-26$ & 3,061716 \\
\hline
\end{tabular}

Tabela 7 - Análise ANOVA - Resistência à compressão das AAMN e AMR

\begin{tabular}{|c|c|c|c|c|c|}
\hline Fonte da variação & SQ & MQ & $\mathbf{F}$ & P-valor & F crítico \\
\hline Resistência à compressão & 3725132 & 1862566 & 181,179 & $2,5 \mathrm{E}-51$ & 3,027783 \\
\hline $\begin{array}{l}\mathrm{SQ}=\text { soma dos quadrados; } \\
\mathrm{MQ}=\text { média dos quadrados; } \\
\mathrm{F}=\text { parâmetro de Fisher para } \\
\mathrm{p} \text {-valor= probabilidade de sig } \\
\mathrm{F} \text { crítico = parâmetro de Fishe }\end{array}$ & $\begin{array}{l}\text { te de sig } \\
\text { ância; e }\end{array}$ & $\begin{array}{l}\text { cância; } \\
\text { este de }\end{array}$ & & & \\
\hline
\end{tabular}

Tabela 8 - Teste de Duncan - Resistência à tração na flexão das AAMN e AAMR

\begin{tabular}{l|c|c|c|c|c}
\hline Argamassa & $\begin{array}{c}\text { Resist. à tração na } \\
\text { flexão (MPa) }\end{array}$ & Grupo 1 & Grupo 2 & Grupo 3 & Grupo 4 \\
\hline AAMN & 2,05 & $\mathrm{X}$ & $\mathrm{X}$ & & \\
AAMR25\% & 1,92 & & & $\mathrm{X}$ & \\
AAMR50\% & 1,67 & & & & $\mathrm{X}$ \\
AAMR10\% & 1,21 & & & \\
\hline
\end{tabular}

Tabela 9 - Teste de Duncan - Resistência à compressão das AAMN e AAMR

\begin{tabular}{l|c|c|c|c|c}
\hline \multicolumn{1}{c|}{ Argamassa } & $\begin{array}{c}\text { Resist. à } \\
\text { compressão (MPa) }\end{array}$ & Grupo 1 & Grupo 2 & Grupo 3 & Grupo 4 \\
\hline AAMN & 6,81 & $\mathrm{X}$ & & & \\
AAMR25\% & 5,84 & & $\mathrm{X}$ & & \\
AAMR50\% & 5,11 & & & $\mathrm{X}$ & $\mathrm{X}$ \\
AAMR100\% & 3,61 & & & \\
\hline
\end{tabular}

Tabela 10 - Módulo de elasticidade dinâmico

\begin{tabular}{c|c|c|c|c}
\hline Ensaio & AAMN & AAMR25\% & AAMR50\% & AAMR100\% \\
\hline Módulo de elasticidade dinâmico $(\mathrm{GPa})$ & 11,47 & 10,27 & 8,86 & 5,76 \\
\hline
\end{tabular}

314 Santana, T. da S.; Pereira, C. H. de A. F. 
De acordo com classificação das argamassas quanto ao módulo de elasticidade do CSTB (CENTRE..., 1993), as AAMN, AAMR25\% eAAMR50\% se enquadraram na classe E4, visto que apresentaram valores de módulo de elasticidade entre 7,5 GPa e 14,0 GPa. Já a AAR100\% se enquadrou na classe E3 (5,0 GPa a 10,0 $\mathrm{GPa})$.

Os valores obtidos são próximos aos determinados por Oliveira (2017), a qual, na avaliação de uma argamassa com areia natural (traço 1:6, em massa) e a combinação de 0,4\% de AEH e 0,2\% de IAR, obteve um módulo de elasticidade de 9,39 GPa por meio da determinação do módulo de elasticidade dinâmico pelo método de excitação por impulso. Macioski et al. (2015), pelo ensaio de módulo de elasticidade dinâmico por propagação de ondas, obtiveram módulos entre 8,8 GPa e 15,38 GPa para argamassas estabilizadas.

\section{Conclusão}

Por meio das avaliações realizadas, concluiu-se que as argamassas com AMN e AMR apresentaram comportamento semelhante no estado fresco, como evidenciado pelos resultados dos ensaios de índice de consistência e penetração de cone. Essa semelhança se deve à fixação da faixa de consistência ao início e ao final do tempo de estabilização das argamassas.

Porém, verificou-se que as argamassas se diferenciaram quanto à massa específica, visto que ocorreu redução da densidade e aumento do teor de ar incorporado com o aumento do teor de substituição dos agregados.

Já no estado endurecido, constatou-se redução das propriedades mecânicas e do módulo de elasticidade, assim como aumento do coeficiente de capilaridade com o aumento dos teores de substituição.

Apesar dessa variação, no estado enrudecido se observou que as AAMN e AAMR25\% se demonstraram semelhantes, visto que se enquadraram nas mesmas classes da NBR 13281 (ABNT, 2005e) quanto à resistência à tração na flexão e à compressão, e mesma classe quanto ao módulo de elasticidade segundo o CSTB (CENTRE...,1993).

Dessa forma, neste estudo a substituição de $25 \%$ de AMN por AMR se mostrou como um teor viável, nos aspectos avaliados, para produção de argamassas estabilizadas de revestimento, pois apresentou um comportamento semelhante ao da AAMN.

\section{Referências}

AMERICAN CONCRETE INSTITUTE. ACI 212.3R-16: report on chemical admixtures for concrete. Farmington Hills, 2016.

AMERICAN SOCIETY FOR TESTING AND MATERIALS. C-780: standard test method for preconstruction and construction evaluation of mortars for plain and reinforced unit masonry. Pennsylvania, 2014.

AMERICAN SOCYETY FOR TESTING END MATERIALS.E-1876: standard test method for dynamic young's modulus, shear modulus, and poisson's ratio by impulse excitation of vibration. Pennsylvania, 2009.

ASSOCIAÇÃO BRASILEIRA DE NORMAS TÉCNICAS. NBR 13276: argamassa para assentamento e revestimento de paredes e tetos: preparo da mistura e determinação do índice de consistência. Rio de Janeiro, 2016.

ASSOCIAÇÃO BRASILEIRA DE NORMAS TÉCNICAS. NBR 13278: argamassa para assentamento e revestimento de paredes e tetos: determinação da densidade de massa e do teor de ar incorporado. Rio de Janeiro, 2005a.

ASSOCIAÇÃO BRASILEIRA DE NORMAS TÉCNICAS. NBR 13277: argamassa para assentamento e revestimento de paredes e tetos: determinação da retenção de água. Rio de Janeiro, 2005b.

ASSOCIAÇÃO BRASILEIRA DE NORMAS TÉCNICAS. NBR 13280: argamassa para assentamento e revestimento de paredes e tetos: determinação da densidade de massa aparente no estado endurecido. Rio de Janeiro, 2005c.

ASSOCIAÇÃO BRASILEIRA DE NORMAS TÉCNICAS. NBR 13279: argamassa para assentamento e revestimento de paredes e tetos: determinação da resistência à tração na flexão e à compressão. Rio de Janeiro, 2005d. 
ASSOCIAÇÃO BRASILEIRA DE NORMAS TÉCNICAS. NBR 13281: argamassa para assentamento e revestimento de paredes e tetos: requisitos. Rio de Janeiro, 2005e.

ASSOCIAÇÃO BRASILEIRA DE NORMAS TÉCNICAS. NBR 15259: argamassa para assentamento e revestimento de paredes e tetos: determinação da absorção de água por capilaridade e do coeficiente de capilaridade. Rio de Janeiro, $2005 \mathrm{f}$.

ASSOCIAÇÃO BRASILEIRA DE NORMAS TÉCNICAS. NBR 15116: agregados reciclados de resíduos sólidos da construção civil: utilização em pavimentação e preparo de concreto sem função estrutural: requisitos. Rio de Janeiro, 2004.

ASSOCIAÇÃO BRASILEIRA DE NORMAS TÉCNICAS. NBR 7218: agregados: determinação do teor de argila em torrões e materiais friáveis. Rio de Janeiro, 2010.

ASSOCIAÇÃO BRASILEIRA DE NORMAS TÉCNICAS. NBR 9776: agregados: determinação da massa específica de agregados miúdos por meio do frasco Chapman: método de ensaio. Rio de Janeiro, 1987.

ASSOCIAÇÃO BRASILEIRA DE NORMAS TÉCNICAS. NBR 9917: agregados para concreto: determinação de sais, cloretos e sulfatos solúveis. Rio de Janeiro, 2009c.

ASSOCIAÇÃO BRASILEIRA DE NORMAS TÉCNICAS. NBR 10908: aditivos para argamassa e concreto: ensaios de caracterização. Rio de Janeiro, 2008.

ASSOCIAÇÃO BRASILEIRA DE NORMAS TÉCNICAS. NBR NM 23: cimento Portland e outros materiais em pó: determinação da massa específica. Rio de Janeiro, 2009a.

ASSOCIAÇÃO BRASILEIRA DE NORMAS TÉCNICAS. NBR NM 248: agregados: determinação da composição granulométrica. Rio de Janeiro, 2003b.

ASSOCIAÇÃO BRASILEIRA DE NORMAS TÉCNICAS. NBR NM 30: agregados miúdos: determinação da absorção de água. Rio de Janeiro, 2000.

ASSOCIAÇÃO BRASILEIRA DE NORMAS TÉCNICAS. NBR NM 46: agregados: determinação do material fino que passa através da peneira 75 um, por lavagem. Rio de Janeiro, 2003a.

ASSOCIAÇÃO BRASILEIRA DE NORMAS TÉCNICAS. NBR NM 65: cimento Portland: determinação do tempo de pega. Rio de Janeiro, 2009b.

BAUER, E. et al.Requisitos das argamassas estabilizadas para revestimento. In: SIMPÓSIO BRASILEIRO DE TECNOLOGIA DAS ARGAMASSAS, 11., Porto Alegre, 2015. Anais [...] Porto Alegre, 2015.

BRAGA, M.; DE BRITO, J.; VEIGA, R. Incorporation of fine concrete aggregates in mortars. Construction and Building Materials, v. 36, p. 960-968, 2012.

BUYLE-BODIN, F.; HADJIEVA-ZAHARIEVA, R. Influence of industrially produced recycled aggregates on flow properties of concrete.Materials and Structures/Materiauxet Constructions, v. 35, p. 504-509, 2002.

CARASEK, H. et al. Estudo e avaliação de agregados reciclados de resíduo de construção e demolição para argamassas de assentamento e de revestimento. Cerâmica, v.64, n. 370, p. 288-300, jun. 2018.

CASALI, J. M. et al. Avaliação das propriedades do estado fresco e endurecido da argamassa estabilizada para assentamento e revestimento. In: SIMPÓSIO BRASILEIRO DE ARGAMASSAS, 9., Belo Horizonte, 2011. Anais [...] Belo Horizonte, 2011.

CENTRE SCIENTIFIQUE ET TECHNIQUE DU BATIMENT. Certification CSTB desenduitsmonocouchesd'imperméabilisation - Cahier MERUC.Livraison 341, cahier 2669-3, 1993.

CONSELHO NACIONAL DO MEIO AMBIENTE. Resolução No 307 de 5 de julho de 2002.Diário Oficial da República Federativa do Brasil, Brasília, DF, n. 136, de 16 de julho de 2002.

CONTRERAS, M. et al. Arecycling of construction and demolition waste for producing new construction material (Brazil case-study). Construction and Building Materials, v. 123, p. 594-600, 2016.

CORINALDESI, V.; MORICONI, G. Behaviour of cementitious mortars containing different kinds of recycled aggregate. Construction and Building Materials, v. 23 p. 289-294, 2009.

316 Santana, T. da S.; Pereira, C. H. de A. F. 
CORRÊA, C. A. R.; RÊGO, J. H.; FEITOSA, C. H. Minucious characterization of granulometric fractions of two fine recycled aggregate. In: INTERNATIONAL CONFERENCE PROGRESS OF RECYCLING IN THE BUILT ENVIRONMENT, 4., Lisbon, 2018. Proceedings [...] Lisbon, 2018.

DIMITRIOU, G.; SAVVA, P.; PETROU, M. F. Enhancing mechanical and durability properties of recycled aggregate concrete. Construction and Building Materials, v. 158, p. 228-235, 2018.

EVANGELISTA, L. et al.Physical, chemical and mineralogical properties of fine recycled aggregates made from concrete waste.Construction and Building Materials, v. 86, p. 178-188, 2015.

FAN, C. C. et al. The effects of different fine recycled concrete aggregates on the properties of mortar.

Materials, v. 8, n. 5, p. 2658-2672, May 2015.

FUMOTO, T.; YAMADA, M. Influence of the quality of recycled fine aggregate on properties of concrete.

Memoirs of the Faculty of Engineering, Osaka, v. 43, p. 97-103, 2002.

JIMÉNEZ, J. R. et al. Use of fine recycled aggregates from ceramic waste in masonry mortar manufacturing. Construction and Building Materials, v. 40, p. 679-690, Mar. 2013.

LEDESMA, E. F. et al. A proposal for the maximum use of recycled concrete sand in masonry mortar design. Materiales de Construcción, v. 66, n. 321, p. e075, 2016.

LEITE, M. B. Avaliação de propriedades mecânicas de concretos produzidos com agregados reciclados de resíduos de construção e demolição. Porto Alegre, 2001. Tese (Doutorado em Engenharia Civil) Programa de Pós-Graduação em Engenharia Civil, Universidade Federal do Rio Grande do Sul, Porto Alegre, 2001.

LEITE, M. B. et al. Utilização de agregado reciclado de concreto para produção de argamassas. In: VIII ENCONTRO NACIONAL DE TECNOLOGIA DO AMBIENTE CONSTRUÍDO, 8., Porto Alegre, 2000.Anais [...]Porto Alegre, 2000.

LLATAS, C. Methods for estimating construction and demolition (C\&D) waste. In: PACHECO-TORGAL, F. et al. (ed.). Handbook of recycled concrete and demolition waste. Cambridge: Woodhead, 2013.

LOFTH, S. et al. Performance of recycled aggregate concrete based on a new concrete recycling technology. Construction and Building Materials, v. 95, p. 243-256, 2015.

MACIOSKI, G.et al. Caracterização de argamassas estabilizadas submetidas à sucção de substrato poroso. In: SIMPÓSIO BRASILEIRO DE TECNOLOGIA DAS ARGAMASSAS, 11., Porto Alegre, 2015. Anais [...] Porto Alegre, 2015.

MARTÍNEZ, I. et al. A comparative analysis of the properties of recycled and natural aggregate in masonry mortars.ConstructionandBuildingMaterials, v. 49, p. 384-392, 2013.

OLIVEIRA, V. C. Estudo comportamental da formulação, dos requisitos e das propriedades das argamassas estabilizadas de revestimento. Brasília, 2017. Dissertação (Mestrado em Engenharia Civil) Programa de Pós-Graduação em Estruturas e Construção Civil, Universidade de Brasília, Brasília, 2017.

PAOLINI, M.; KHURANA R. Admixtures for recycling of waste concrete. Cement and Concrete Composites, v. 20, p. 221-229, 1998.

PAZ, D. H.; LAFAYETTE, K. P. Forecasting of construction and demolition waste in Brazil. Waste Management \&Research, v. 34, p. 708-716, 2016.

PEDROZO, R. F. E. Influência da substituição do agregado miúdo natural por agregado reciclado fino em propriedades de argamassas e concretos. Florianópolis, 2008. Dissertação (Mestrado em Engenharia Civil) - Programa de Pós-Graduação em Engenharia Civil, Universidade Federal de Santa Catarina, Florianópolis, 2008.

PIMENTEL, L. L. et al. Argamassa com areia proveniente da britagem de resíduo de construção civil: avaliação de características físicas e mecânicas. Revista Matéria, v. 23, n. 1, mar. 2018.

RIXON, R.; MAILVAGANAM, N. Chemical admixtures for concrete. 3. ed. London: E\&FN Spon, 1999.

SAMIE, R. R. et al. Properties of cement-lime mortars vs. cement mortars containing recycled concrete aggregates. ConstructionandBuildingMaterials, v. 84, 1, p. 84-94, jun. 2015. 
SANTANA, T. S. Avaliação da influência da utilização de agregado miúdo reciclado em argamassas estabilizadas. Brasília, 2018. Dissertação (Mestrado em Engenharia Civil) - Programa de Pós-Graduação em Estruturas e Construção Civil, Universidade de Brasília, Brasília, 2018.

SANTOS, W. F. Tratamento superficial dos agregados reciclados de RCD com hidrofugantes. São Paulo, 2016. Dissertação (Mestrado em Ciências do Ambiente) - Escola Politécnica, Universidade de São Paulo, São Paulo, 2016.

SOLYMAN, M. Classification of recycled sands and their applications as fine aggregates for concrete and bituminous mixtures. Kassel, 2005. Dissertation - FachbereichBauingenieurwesen der Universität Kassel, Kassel, 2005.

TREVISOL JUNIOR, L. A. Estudo comparativo entre as argamassas: estabilizada dosada em central, industrializada e produzida em obra por meio de ensaios físicos nos estados fresco e endurecido. Curitiba, 2015. Dissertação (Mestrado em Engenharia Civil) - Instituto de Engenharia do Parana, Curitiba, 2015.

\section{Thiago da Silva Santana}

Departamento de Engenharia | Civil e Ambiental | Universidade de Brasília | Campus Universitário Darcy Ribeiro, Asa Norte | Brasília -DF - Brasil | CEP 70910-900 | Tel.: (61) 98550-5019 | E-mail: santana.tsilva@gmail.com

Cláudio Henrique de Almeida Feitosa Pereira

Departamento de EngenhariaCivil e Ambiental | Universidade de Brasília | Tel.: (61) 98115-8783 | E-mail: claudiopereira@unb.br

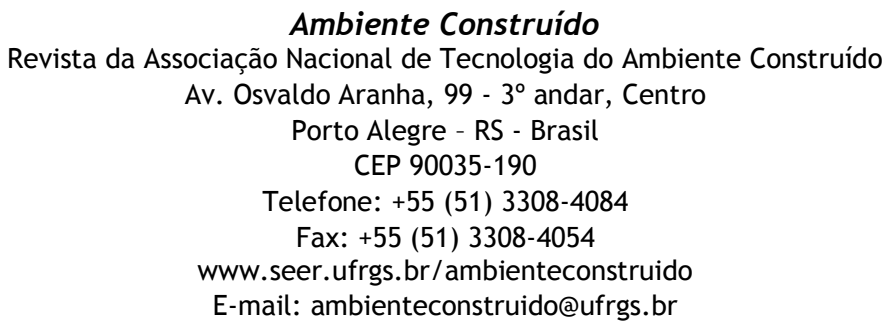

